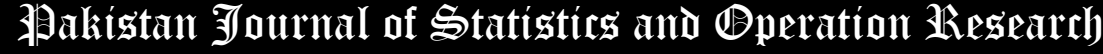

\section{A Novel Generator of Continuous Probability Distributions for the Asymmetric Left-skewed Bimodal Real-life Data with Properties and Copulas}

\author{
Wahid A. M. Shehata ${ }^{1}$ Haitham M. Yousof ${ }^{2, *}$, Mohamed Aboraya ${ }^{3}$ \\ * Corresponding Author
}

1. Department of Mathematics, Statistics and Insurance, Faculty of Business, Ain Shams University, Egypt. Email: wahid75maher@yahoo.com

2. Department of Statistics, Mathematics and Insurance, Benha University, Benha 13518, Egypt, haitham.yousof@fcom.bu.edu.eg

3. Department of Applied, Mathematical and Actuarial Statistics, Faculty of Commerce, Damietta University, Damietta, Egypt. E-mail: mohamedaboraya17@gmail.com

\begin{abstract}
This paper presents a novel two-parameter $\mathrm{G}$ family of distributions. Relevant statistical properties such as the ordinary moments, incomplete moments and moment generating function are derived. Using common copulas, some new bivariate type $\mathrm{G}$ families are derived. Special attention is devoted to the standard exponential base line model. The density of the new exponential extension can be "asymmetric and right skewed shape" with no peak, "asymmetric right skewed shape" with one peak, "symmetric shape" and "asymmetric left skewed shape" with one peak. The hazard rate of the new exponential distribution can be "increasing", "U-shape", "decreasing" and "Jshape". The usefulness and flexibility of the new family is illustrated by means of two applications to real data sets. The new family is compared with many common $\mathrm{G}$ families in modeling relief times and survival times data sets.
\end{abstract}

Key Words: Poisson Family; Generalized Weibull Family; compounding; Farlie-Gumbel-Morgenstern; Clayton copula; Modeling; Lomax distribution; Ali-Mikhail-Haq copula.

Mathematical Subject Classification: 62N01; 62N02; 62E10.

\section{Introduction and motivation}

Statistical literature contains various $\mathrm{G}$ families of distributions which were generated either by compounding common existing $\mathrm{G}$ families or by adding one (or more) parameters to the existing $\mathrm{G}$ families. These novel families were employed for modeling real data in many applied studies such as engineering, insurance, demography, medicine, econometrics, biology, environmental sciences and forecasting approaches see, for example, see Yousof et al. (2015) (transmuted generalized exponentiated family), Merovci et al. (2017) (exponentiated transmuted family), Brito et al. (2017) (Topp Leone odd log-logistic family), Hamedani et al. (2017) (type I general exponential family), Yousof et al. (2017a) (Burr X family), Cordeiro et al. (2018) (Burr XII family), Korkmaz et al. (2018a) (exponential-Lindley odd log-logistic family), Hamedani et al. (2018) (an extended G family), Yousof et al. (2018b) (Burr Hatke family), Hamedani et al. (2019) ( type II general exponential family), Nascimento et al. (2019) (odd Nadarajah-Haghighi family) and Karamikabir et al. (2020) (Weibull Topp Leone generated family), among others. In this paper we propose and study a new family of distributions using the zero truncated Poisson (ZTP) distribution with a strong physical motivation. 
Suppose that a system has $N$ subsystems functioning independently at a given time where $N$ has ZTP distribution with parameter $\lambda=1$. It is the conditional probability distribution of a Poisson-distributed random variable (RV), given that the value of the RV is not zero. The probability mass function (PMF) of $N$ is given by

$$
P(N=n)=[\exp (-1)] /\left.\{n ![-\exp (-1)+1]\}\right|_{(n=1,2, \ldots)} .
$$

Suppose that the failure time of each subsystem has the generalized Weibull generator (GW-G) defined by Cordeiro et al. (2017). The cumulative distribution function (CDF) of the GW-G is given as

$$
\mathbf{H}_{a, b, \underline{v}}(z)=\left.\left\{1-\exp \left[-\mathcal{W}_{b, \underline{v}}(z)\right]\right\}^{a}\right|_{z \in \mathcal{R}}
$$

where the function $\boldsymbol{W}_{b, \underline{v}}(z)=\left.\left[\mathbf{H}_{\underline{\underline{v}}}(z) / \overline{\mathbf{H}}_{\underline{\underline{v}}}(z)\right]^{b}\right|_{z \in \mathcal{R}}$ refers to the odd ration function (ORF), $\mathbf{H}_{\underline{\underline{v}}}(z)$ refers to the base line CDF with parameters vector $\underline{\mathcal{v}}, \overline{\mathbf{H}}_{\underline{v}}(z)=1-\mathbf{H}_{\underline{v}}(z)$ refers to the base line model survival function (SF), $h_{\underline{v}}(z)=d \mathbf{H}_{\underline{v}}(z) / d z$ is the base line probability density function (PDF) and $a, b>0$ is a shape parameters.

Staying in (1) and for $b=2$, the GW-G reduces to generalized Rayleigh G (ER-G) (Yousof et al. (2017a)) which is also called the Burr X (BX-G). Let $Y_{i}$ denote the failure time of the ith subsystem and let

Then the conditional CDF of $Z$ given $N$ is

$$
Z=\min \left\{Y_{1}, Y_{2}, \cdots, Y_{N}\right\}
$$

$$
F(z)=1-\operatorname{Pr}\left(Z>\left.z\right|_{N}\right)=1-\left[1-\mathbf{H}_{a, b, \underline{v}}(z)\right]^{N} .
$$

Therefore, the unconditional CDF of the QPGW-G density function can be expressed as described in Ramos et al. (2015), Aryal and Yousof (2017), Yousof et al. (2018a) and Yousof et al. (2020), among others.

The proposed family is most conveniently specified in terms of the ZTP generator applied to the generalized WeibullG class. By inserting (1) in equation (2), the CDF of the quasi-Poisson generalized Weibull-G (QPGW-G) family is given by

$$
F_{\underline{\Phi}}(z)=\left.\frac{1}{1-\exp (-1)}\left[1-\exp \left(-\left\{1-\exp \left[-\boldsymbol{w}_{b, \underline{v}}(z)\right]\right\}^{a}\right)\right]\right|_{z \in \mathcal{R}}
$$

where $\underline{\Phi}=(a, b, \underline{\mathcal{v}})$ is the parameter vector of the QPGW-G family.

The following special cases can be considered:

$i$. For $b=1$, the QPGW-G family reduces to the quasi-Poisson generalized exponential-G family.

ii. $\quad$ For $b=2$, the QPGW-G family reduces to quasi-Poisson generalized Rayleigh-G family.

iii. For $a=1$, the QPGW-G family reduces to the quasi-Poisson Weibull-G family (Yousof et al. (2020)).

iv. For $a=b=1$, the QPGW-G family reduces to quasi-Poisson exponential-G family.

v. For $b=2$ and $a=1$, the QPGW-G family reduces to the reduced quasi-Poisson exponential-G family (Yousof et al. (2020)).

The PDF of the QPGW-G family can then be expressed as

$$
f_{\underline{\Phi}}(z)=\left.a b \frac{\boldsymbol{h}_{\underline{v}}(z) \mathbf{H}_{\underline{v}}(z)^{b-1} \exp \left[-\boldsymbol{W}_{b, \underline{v}}(z)\right] \exp \left(-\left\{1-\exp \left[-\boldsymbol{w}_{b, \underline{v}}(z)\right]\right\}^{a}\right)}{[1-\exp (-1)] \overline{\mathbf{H}}_{\underline{v}}(z)^{b+1}\left\{1-\exp \left[-\boldsymbol{W}_{b, \underline{v}}(z)\right]\right\}^{1-a}}\right|_{z \in \mathcal{R}}
$$

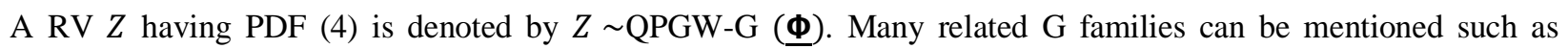
exponentiated generalized Poisson family (Aryal and Yousof (2017)), Marshall-Olkin generalized Poisson family (Korkmaz et al. (2018b)), Weibull Poisson family (Yousof et al. (2020)), Poisson Topp Leone family (Merovci et al. (2020)) and Poisson generalized exponential family (El-Morshedy et al. (2021)).

On the other hand, many common copulas are employed for deriving new bivariate type QPGW-G families such as "Farlie-Gumbel-Morgenstern (FGM) copula", "Clayton copula", and "Renyi's entropy copula". Fisher (1997) provided two major justifications as to why copulas are useful and of interest to statisticians. Firstly, as a way for studying scale-free measures of dependence. 
Secondly, as a starting point for constructing new bivariate $\mathrm{G}$ families of distributions. Precisely, copulas are an important part of the study of dependence between two variables since they allow us to separate the effect of dependence from the effects of the marginal distributions. Further future articles could be allocated to study the new bivariate type $\mathrm{G}$ families.

We are motivated to present the new family since it could be useful in modeling variable real-life data as illustrated below:

i. The real data which have an " increasing failure rate " (see Figures 3 and 5 (bottom left panels)).

ii. The real data which have some outliers (see Figures 3 and 5 (top right and top left panels)).

iii. The real data sets which their Kernel density estimation are asymmetric and bimodal with right tail (see Figures 3 and 5 (bottom right plots)).

iv. The real data which their PDF can be "asymmetric and right skewed shape" with no peak, "asymmetric right skewed shape" with one peak, "symmetric shape" and "asymmetric left skewed shape" with one peak (see Figure 1).

v. The real-life datasets which their HRF can be "increasing", "U-shape", "decreasing" and "J-shape" (see Figure 2).

Additionally, in modeling the relief times data and the survival times of the aircraft windshield data the novel family based on the quasi Poisson generalized Weibull- exponential model is better than many other common exponential extensions such as the Marshall-Olkin exponential, Moment exponential, the Burr-Hatke exponential, Generalized Marshall-Olkin exponential, the odd Lindley exponential, Beta exponential, Kumaraswamy Marshall-Olkin exponential, Marshall-Olkin Kumaraswamy exponential, the Burr X exponential, Kumaraswamy exponential and standard exponential model under the eight criteria called Anderson-Darling Criteria, Akaike Information Criteria, Cramér-Von Mises Criteria, Hannan-Quinn Information Criteria, Bayesian Information Criteria, Consistent Akaike Information Criteria, Kolmogorov-Smirnov (KS) statistic test and its corresponding P-value.

\section{Copulas for the QPGW-G family}

In this Section, we derive some new bivariate QPGW-G (BQPGW-G) type model using the FGM copula (Morgenstern (1956), Gumbel (1960), Gumbel (1960)) and Johnson and Kotz (1977)), "Clayton copula", the "Ali-Mikhail-Haq" copula (Ali et al. (1978)) and "Renyi's entropy" (Pougaza and Djafari (2011)). The Multivariate QPGW-G (MvQPGW-G) type is also presented.

Recently, many new articles have been studied some copulas such as Ali et al. (2021a and b), Aboraya, M. (2021a), El-Morshedy et al. (2021). However, future works may be allocated to study these new models.

\subsection{BQPGW-G type via FGM copula}

Consider the joint CDF of the FGM family where

$$
\mathcal{C}_{\varsigma}(\mathcal{P}, \mathcal{Q})=\mathcal{P} Q(1+\varsigma \overline{\mathcal{P} Q})
$$

where the continuous marginal function $\mathcal{P} \in(0,1)$ and $\mathcal{Q} \in(0,1)$. The parameter $\varsigma \in[-1,1]$ is a dependence parameter.

For every $\mathcal{C}_{\zeta}(\mathcal{P}, 0)=\mathcal{C}_{\zeta}(0, \mathcal{Q})=\left.0\right|_{(\mathcal{P}, \mathcal{Q} \in(0,1))}$, which is "grounded minimum" and $\mathcal{C}_{\zeta}(\mathcal{P}, 1)=\mathcal{P}$ and $\mathcal{C}_{\zeta}(1, \mathcal{Q})=\mathcal{Q}$ which is "grounded maximum". Then, setting

and

$$
\overline{\mathcal{P}}=\overline{\mathcal{P}}_{\underline{\Phi}_{1}}=1-\left.\frac{1}{1-\exp (-1)}\left[1-\exp \left(-\left\{1-\exp \left[-\mathcal{W}_{b_{1}, \underline{v}}\left(z_{1}\right)\right]\right\}^{a_{1}}\right)\right]\right|_{\underline{\Phi}_{1}>0}
$$

Then, we have

$$
\overline{\mathcal{Q}}=\overline{\mathcal{Q}}_{{\underline{\Phi_{2}}}_{2}}=1-\left.\frac{1}{1-\exp (-1)}\left[1-\exp \left(-\left\{1-\exp \left[-\mathfrak{w}_{b_{2}, \underline{v}}\left(z_{2}\right)\right]\right\}^{a_{2}}\right)\right]\right|_{\underline{\Phi}_{2}>0}
$$

$$
\begin{aligned}
F\left(z_{1}, z_{2}\right)=\mathcal{C}\left(F_{\underline{\Phi}_{1}}\left(z_{1}\right),\right. & \left.F_{\underline{\Phi}_{2}}\left(z_{2}\right)\right)=\frac{1}{[1-\exp (-1)]^{2}}\left[1-\exp \left(-\left\{1-\exp \left[-\mathfrak{W}_{b_{1}, \underline{v}}\left(z_{1}\right)\right]\right\}^{a_{1}}\right)\right] \\
& \times\left[1-\exp \left(-\left\{1-\exp \left[-\mathfrak{W}_{b_{2}, \underline{v}}\left(z_{2}\right)\right]\right\}^{a_{2}}\right)\right]
\end{aligned}
$$


The joint PDF (J-CDF) can be derived from

$$
\times\left[1+\varsigma\left(\begin{array}{l}
\left\{1-\frac{1}{1-\exp (-1)}\left[1-\exp \left(-\left\{1-\exp \left[-\mathcal{W}_{b_{1}, \underline{v}}\left(z_{1}\right)\right]\right\}^{a_{1}}\right)\right]\right\} \\
\left\{1-\frac{1}{1-\exp (-1)}\left[1-\exp \left(-\left\{1-\exp \left[-\mathcal{W}_{b_{2}, \underline{v}}\left(z_{2}\right)\right]\right\}^{a_{2}}\right)\right]\right\}
\end{array}\right)\right]
$$

$$
c_{\zeta}(\mathcal{P}, \mathcal{Q})=1+\left.\varsigma \mathcal{P}^{*} Q^{*}\right|_{\left(\mathcal{P}^{*}=1-2 \mathcal{P} \text { and } Q^{*}=1-2 Q\right)} .
$$

\subsection{BQPGW-G type via Clayton copula}

The Clayton copula can be considered as

$$
\mathcal{C}\left(\mathcal{P}_{1}, \mathcal{P}_{2}\right)=\left.\left(\mathcal{P}_{1}^{-\varsigma}+\mathcal{P}_{2}^{-\varsigma}-1\right)^{-\frac{1}{\varsigma}}\right|_{\varsigma \in[0, \infty]} .
$$

Let us assume that $X \sim \mathrm{QPGW}-\mathrm{G}\left(a_{1}, b_{1}\right)$ and $Y \sim \mathrm{QPGW}-\mathrm{G}\left(a_{2}, b_{2}\right)$. Then, setting

and

$$
\mathcal{P}_{1}=\mathcal{P}(x)=\left.\frac{1}{1-\exp (-1)}\left[1-\exp \left(-\left\{1-\exp \left[-\mathcal{W}_{b_{1}, \underline{v}}(x)\right]\right\}^{a_{1}}\right)\right]\right|_{\underline{\Phi}_{1}>0},
$$

Then, the BQPGW-G type distribution can be derived as

$$
\mathcal{P}_{2}=\mathcal{P}(z)=\left.\frac{1}{1-\exp (-1)}\left[1-\exp \left(-\left\{1-\exp \left[-\mathfrak{w}_{b_{2}, \underline{v}}(z)\right]\right\}^{a_{2}}\right)\right]\right|_{\underline{\Phi}_{2}>0},
$$

$$
F(x, z)=\mathcal{C}\left(F_{\underline{\Phi}_{1}}(x), F_{\underline{\Phi}_{2}}(z)\right)=\left[\begin{array}{c}
\left(\frac{1}{1-\exp (-1)}\left[1-\exp \left(-\left\{1-\exp \left[-\mathcal{W}_{b_{1}, \underline{v}}(x)\right]\right\}^{a_{1}}\right)\right]\right)^{-\varsigma} \\
+\left(\frac{1}{1-\exp (-1)}\left[1-\exp \left(-\left\{1-\exp \left[-\mathcal{W}_{b_{2}, \underline{v}}(y)\right]\right\}^{a_{2}}\right)\right]\right)^{-\varsigma} \\
-1
\end{array}\right]^{-\frac{1}{\varsigma}} .
$$

\subsection{BQPGW-G type via Renyi's entropy}

Consider theorem of Pougaza and Djafari (2011) where

then, the associated CDF of the BQPGW-G will be

$$
\mathcal{C}(\mathcal{P}, \mathcal{Q})=z_{2} \mathcal{P}+z_{1} \mathcal{Q}-z_{1} z_{2}
$$

$$
\begin{aligned}
& \mathcal{C}\left(z_{1}, z_{2}\right)=R\left(F_{\underline{\Theta}_{1}}\left(z_{1}\right), F_{\underline{\Theta}_{2}}\left(z_{1}\right)\right)=-z_{1} z_{2} \\
& +z_{2}\left\{\frac{1}{1-\exp (-1)}\left[1-\exp \left(-\left\{1-\exp \left[-\mathcal{W}_{b_{1}, \underline{v}}\left(z_{1}\right)\right]\right\}^{a_{1}}\right)\right]\right\} \\
& +z_{1}\left\{\frac{1}{1-\exp (-1)}\left[1-\exp \left(-\left\{1-\exp \left[-\mathcal{W}_{b_{2}, \underline{v}}\left(z_{2}\right)\right]\right\}^{a_{2}}\right)\right]\right\} .
\end{aligned}
$$

\subsection{BQPGW-G type via Ali-Mikhail-Haq copula}

Under the stronger Lipschitz condition, the joint CDF of the Archimedean Ali-Mikhail-Haq copula can expressed as

$$
\mathcal{C}(\mathcal{P}, \mathcal{Q})=\left.\frac{\mathcal{P} Q}{1-\varsigma \overline{\mathcal{P} Q}}\right|_{\varsigma \in(-1,1)}
$$

the corresponding J-PDF of the Archimedean Ali-Mikhail-Haq copula can expressed as

$$
c(\mathcal{P}, \mathcal{Q})=\left.\frac{1}{[1-\varsigma \overline{\mathcal{P} Q}]^{2}}\left(1-\varsigma+2 \varsigma \frac{\mathcal{P} Q}{1-\varsigma \overline{\mathcal{P} Q}}\right)\right|_{\varsigma \in(-1,1)},
$$

then for any $Z_{1} \sim$ QPGW-G $\left(a_{1}, b_{1}\right)$ and $Z_{2} \sim$ QPGW-G $\left(a_{2}, b_{2}\right)$ we have

The J-PDF is straightforward then omitted.

$$
\mathcal{C}\left(z_{1}, z_{2}\right)=\left.\frac{\left\{\begin{array}{c}
\frac{1}{[1-\exp (-1)]^{2}}\left[1-\exp \left(-\left\{1-\exp \left[-\mathfrak{w}_{b_{1}, \underline{v}}\left(z_{1}\right)\right]\right\}^{a_{1}}\right)\right] \\
\times\left[1-\exp \left(-\left\{1-\exp \left[-\mathfrak{W}_{b_{2}, \underline{v}}\left(z_{2}\right)\right]\right\}^{a_{2}}\right)\right]
\end{array}\right\}}{1-\varsigma\left(\begin{array}{c}
\left\{1-\frac{1}{1-\exp (-1)}\left[1-\exp \left(-\left\{1-\exp \left[-\mathfrak{W}_{b_{1}, \underline{v}}\left(z_{1}\right)\right]\right\}^{a_{1}}\right)\right]\right\} \\
\times\left\{1-\frac{1}{1-\exp (-1)}\left[1-\exp \left(-\left\{1-\exp \left[-\mathfrak{W}_{b_{2}, \underline{v}}\left(z_{2}\right)\right]\right\}^{a_{2}}\right)\right]\right\}
\end{array}\right)}\right|_{\varsigma \in(-1,1)} .
$$




\subsection{The MvQPGW-G type}

Following Nelsen (2007) and Balakrishnan and Lai (2009), a straightforward Multivariate QPGW-G $\hbar$-dimensional extension can be derived from

$$
H\left(z_{i}\right)=\left(\sum_{i=1}^{\hbar}\left\{c_{1}^{-1}\left[1-\exp \left(-\left\{1-\exp \left[-\mathcal{W}_{b_{i}, \underline{v}}\left(z_{i}\right)\right]\right\}^{a_{i}}\right)\right]\right\}^{-\varsigma}+1-\hbar\right)^{-\frac{1}{\varsigma}} .
$$

\section{Mathematical Properties}

\subsection{Linear representation}

In this section, we derive a useful linear representation for the QPGW-G density function. Using the power series, we expand the quantity $A(z)$ as

$$
A(z)=\exp \left(-\left\{1-\exp \left[-\mathfrak{w}_{b, \underline{v}}(z)\right]\right\}^{a}\right)=\sum_{\ell=0}^{+\infty} \frac{1}{\ell !}(-1)^{\ell}\left\{1-\exp \left[-\mathfrak{w}_{b, \underline{v}}(z)\right]\right\}^{a \ell} .
$$

Then, the PDF in (4) can be expressed as

$$
f_{\underline{\Phi}}(z)=\frac{a b}{1-\exp (-1)} \sum_{\ell=0}^{+\infty} \frac{(-1)^{\ell} \exp \left[-\mathcal{W}_{b, \underline{v}}(z)\right] \boldsymbol{h}_{\underline{v}}(z)}{\ell ! \overline{\mathbf{H}}_{\underline{\underline{v}}}(z)^{b+1} \mathbf{H}_{\underline{v}}(z)^{-b+1}} \underbrace{\left\{1-\exp \left[-\boldsymbol{W}_{b, \underline{v}}(z)\right]\right\}^{a(\ell+1)-1}}_{B(z)} .
$$

Then, consider the power series

$$
\left(1-\frac{\varsigma_{1}}{\varsigma_{2}}\right)^{1+\varsigma_{3}}=\left.\sum_{i=0}^{+\infty}(-1)^{i} \frac{\Gamma\left(2+\varsigma_{3}\right)}{i ! \Gamma\left(2+\varsigma_{3}-i\right)}\left(\frac{\varsigma_{1}}{\varsigma_{2}}\right)^{i}||_{\varsigma_{2}}\right|_{<1} \text { and } \varsigma_{3}>0 .
$$

Applying (6) to the quantity $B(z)$ in (5), we get

$$
f_{\underline{\Phi}}(z)=a b \frac{\boldsymbol{h}_{\underline{v}}(z) \mathbf{H}_{\underline{\underline{v}}}(z)^{b-1}}{[1-\exp (-1)] \overline{\mathbf{H}}_{\underline{v}}(z)^{b+1}} \sum_{\ell, i=0}^{+\infty} \frac{(-1)^{\ell+i} \Gamma(a(\ell+1))}{i ! \ell ! \Gamma(a(\ell+1)-i)} \underbrace{\exp \left[-(i+1) \boldsymbol{w}_{b, \underline{v}}(z)\right]}_{C(z)} .
$$

Expanding the quantity $C(z)$ in power series, we can write

$$
C(z)=\sum_{p=0}^{+\infty}(-1)^{p} \frac{(i+1)^{p}}{p !} \frac{\mathbf{H}_{\underline{v}}(z)^{p b}}{\overline{\mathbf{H}}_{\underline{\underline{v}}}(z)^{p b}}
$$

Inserting the above expression of $\mathcal{C}_{i}(z)$ in (9), the QPGW-G density reduces to

$$
f_{\underline{\Phi}}(z)=a b \sum_{\ell, i, p=0}^{+\infty} \frac{(-1)^{\ell+p+i} \Gamma(a(\ell+1))(i+1)^{p}}{\ell ! i ! p ![1-\exp (-1)] \Gamma(a(\ell+1)-i)} \frac{\boldsymbol{h}_{\underline{\underline{v}}}(z) \mathbf{H}_{\underline{v}}(z)^{(p+1) b-1}}{\overline{\mathbf{H}}_{\underline{v}}(z)^{(p+1) b+1}} .
$$

Using the generalized binomial expansion to $\left[1-\mathbf{H}_{\underline{\underline{v}}}(z)\right]^{-[(p+1) b+1]}$, we can write

$$
\left[1-\mathbf{H}_{\underline{\xi}}(z)\right]^{-[(p+1) b+1]}=\sum_{q=0}^{+\infty} \frac{\Gamma([p+1] b+q+1)}{q ! \Gamma([p+1] b+1)} \mathbf{H}_{\underline{v}}(z)^{q} .
$$

Inserting (10) in (9), the QPGW-G density can be expressed as an infinite linear combination of exp-G density functions

$$
f_{\underline{\Phi}}(z)=\left.\sum_{p, q=0}^{+\infty} \Upsilon_{p, q} \boldsymbol{h}_{b^{*}}(z)\right|_{b^{*}=[p+1] b+q}
$$

where $\boldsymbol{h}_{b^{*}}(z)=d \mathbf{H}_{b^{*}}(z) / d z=b^{*} \boldsymbol{h}(z) \mathbf{H}_{\underline{v}}(z)^{b^{*}-1}$ is the exp-G PDF with power parameter $b^{*}$ and

$$
r_{p, q}=\frac{a b}{1-\exp (-1)} \sum_{\ell, i=0}^{+\infty} \frac{(-1)^{\ell+p+i}(i+1)^{p} \Gamma(a(\ell+1)) \Gamma\left(b^{*}+1\right)}{\ell ! i ! p ! q ! b^{*} \Gamma(a(\ell+1)-i) \Gamma([p+1] b+1)} \text {. }
$$

Equation (11) reveals that the PDF of QPGW-G family can be expressed as a linear combination of exp-G PDFs. So, several mathematical properties of the new family can be obtained by knowing those of the exp-G distribution. 
Similarly, the CDF of the QPGW-G family can also be expressed as a linear combination of exp-G CDFs given by

$$
F_{\Phi}(z)=\sum_{p, q=0}^{+\infty} r_{p, q} \mathbf{H}_{b^{*}}(z)
$$

where $\mathbf{H}_{b^{*}}(z)$ is the exp-G CDF with power parameter $b^{*}$.

\subsection{Moments}

The $r^{\text {th }}$ moment of $Z$, say $\mu_{r, Z}^{\prime}$, follows from equation (12) as

$$
\mu_{r, Z}^{\prime}=E\left(Z^{r}\right)=\sum_{p, q=0}^{+\infty} r_{p, q} E\left(Y_{b^{*}}^{r}\right)
$$

where $Y_{b^{*}}$ denotes the exp-G RV with power parameter $b^{*}$. The $n$th central moment of $Z$, say $M_{n}$, is given by

$$
M_{n, Z}=E\left(Z-\mu_{1, Z}^{\prime}\right)^{n}=\sum_{r=0}^{n}\left(\begin{array}{l}
n \\
r
\end{array}\right)\left(-\mu_{1, Z}^{\prime}\right)^{n-r} E\left(Z^{r}\right) .
$$

3.3 Moment generating function and the characteristic function

The moment generating function (MGF) of $Z$ can follow from equation (12) as

$$
M_{Z}(t)=\sum_{p, q=0}^{+\infty} r_{p, q} M_{b^{*}}(t)
$$

where $M_{b^{*}}(t)$ is the MGF of $Y_{b^{*}}$ (for $p, q \geq 0$ ). Hence, $M_{Z}(t)$ can be easily obtained from the exp-G generating function. The characteristic function (CF) of $Z$ can be derived from

$$
C_{Z}(i t)=\sum_{p, q=0}^{+\infty} r_{p, q} M_{b^{*}}(i t)
$$

where $M_{b^{*}}(i t)$ is the CF of $Y_{b^{*}}($ for $p, q \geq 0)$ and $i=\sqrt{-1}$.

\subsection{Incomplete moments}

The $s^{\text {th }}$ incomplete moment, say $\boldsymbol{\Delta}_{s, Z}(t)$, of $Z$ can be expressed from (12) as

$$
\boldsymbol{\Delta}_{s, Z}(t)=\int_{-\infty}^{t} z^{s} f(z) d z=\sum_{p, q=0}^{n} r_{p, q} \int_{-\infty}^{t} z^{s} \boldsymbol{h}_{b^{*}}(z) d z .
$$

Clearly, the integral in equation (13) denotes the $s^{\text {th }}$ incomplete moment of $Y_{b^{*}}$.

\subsection{Convex-concave analysis}

Convex probability density functions play a very important role in many areas of mathematics. They are important especially in studying of the "optimization problems" where they are distinguished by several convenient properties. In mathematical analysis, a certain PDF defined on certain n-dimensional interval is called "convex probability density function " if the line between any two points on the graph of the probability density function lies above the graph between the two points.

The PDF in (4) is said to be "concave probability density function" if for any $X_{1} \sim \mathrm{QPGW}-\mathrm{G}\left(\underline{\Phi}_{1}\right)$ and $X_{2} \sim$ QPGW $-\mathrm{G}\left(\underline{\Phi}_{1}\right)$ the probability density function satisfies

$$
f\left(\gamma x_{1}+\bar{\gamma} x_{2}\right) \geq \gamma f_{\Phi_{1}}\left(x_{1}\right)+\left.\bar{\gamma} f_{\Phi_{2}}\left(x_{2}\right)\right|_{0 \leq \gamma \leq 1 \text { and } \bar{\gamma}=1-\gamma}
$$

If the function $f\left(\gamma x_{1}+\bar{\gamma} x_{2}\right)$ is twice differentiable, then if $f^{/ /}\left(\gamma x_{1}+\bar{\gamma} x_{2}\right)<0, \forall x \in \mathbb{R}$, then $f\left(\gamma x_{1}+\bar{\gamma} x_{2}\right)$ is "strictly convex". If $f^{/ /}\left(\gamma x_{1}+\bar{\gamma} x_{2}\right) \leq 0, \forall x \in \mathbb{R}$, then $f\left(\gamma x_{1}+\bar{\gamma} x_{2}\right)$ is "convex".

The PDF in (4) is said to be "convex probability density function" if for any $X_{1} \sim \mathrm{QPGW}-\mathrm{G}\left(\underline{\Phi}_{1}\right)$ and $X_{2} \sim$ QPGW - G $\left(\underline{\Phi}_{1}\right)$ the probability density function satisfies 


$$
f\left(\gamma x_{1}+\bar{\gamma} x_{2}\right) \leq \gamma f_{\Phi_{1}}\left(x_{1}\right)+\left.\bar{\gamma} f_{\Phi_{2}}\left(x_{2}\right)\right|_{0 \leq \gamma \leq 1 \text { and }} \bar{\gamma}=1-\gamma
$$

If the function $f\left(\gamma x_{1}+\bar{\gamma} x_{2}\right)$ is twice differentiable, then if $f^{\prime /}\left(\gamma x_{1}+\bar{\gamma} x_{2}\right)>0, \forall x \in \mathbb{R}$, then $f\left(\gamma x_{1}+\bar{\gamma} x_{2}\right)$ is "strictly convex". If $f^{/ /}\left(\gamma x_{1}+\bar{\gamma} x_{2}\right) \geq 0, \forall x \in \mathbb{R}$, then $f\left(\gamma x_{1}+\bar{\gamma} x_{2}\right)$ is "convex". If $f\left(\gamma x_{1}+\bar{\gamma} x_{2}\right)$ is "convex" and $c$ is a constant, then the function $c f\left(\gamma x_{1}+\bar{\gamma} x_{2}\right)$ is "convex". If $f\left(\gamma x_{1}+\bar{\gamma} x_{2}\right)$ is "convex probability density function", then $\left[c f\left(\gamma x_{1}+\bar{\gamma} x_{2}\right)\right]$ is convex for every $c>0$. If $f\left(\gamma x_{1}+\bar{\gamma} x_{2}\right)$ and $g\left(\gamma x_{1}+\bar{\gamma} x_{2}\right)$ are "convex probability density function" then $\left[f\left(\gamma x_{1}+\bar{\gamma} x_{2}\right)+g\left(\gamma x_{1}+\bar{\gamma} x_{2}\right)\right]$ is also "convex probability density function". If $f\left(\gamma x_{1}+\bar{\gamma} x_{2}\right)$ and $g\left(\gamma x_{1}+\bar{\gamma} x_{2}\right)$ are "convex probability density function" then $\left[f\left(\gamma x_{1}+\bar{\gamma} x_{2}\right) \cdot g\left(\gamma x_{1}+\bar{\gamma} x_{2}\right)\right]$ is also "convex probability density function". If the function $-f\left(\gamma x_{1}+\bar{\gamma} x_{2}\right)$ is "convex probability density function", then the function $f\left(\gamma x_{1}+\bar{\gamma} x_{2}\right)$ is "convex probability density function". If $f\left(\gamma x_{1}+\bar{\gamma} x_{2}\right)$ is "concave probability density function", then $\frac{1}{f\left(\gamma x_{1}+\bar{\gamma} x_{2}\right)}$ is "convex probability density function" if $f(x)>0$. If $f\left(\gamma x_{1}+\bar{\gamma} x_{2}\right)$ is "concave probability density function", $\frac{1}{f\left(\gamma x_{1}+\bar{\gamma} x_{2}\right)}$ is "convex probability density function" if $f(x)<0$. If $f\left(\gamma x_{1}+\bar{\gamma} x_{2}\right)$ is "concave probability density function", $\frac{1}{f\left(\gamma x_{1}+\bar{\gamma} x_{2}\right)}$ is "convex probability density function".

\section{A special case}

In this section, we will focus on the base line exponential distribution. The CDF of the standard exponential model can be expressed as

$$
F_{c}(z)=1-\left.\exp (-c z)\right|_{c>0, z>0}
$$

Based on (3), the CDF of the quasi-Poisson generalized Weibull-exponential (QPGW-E) distribution is can then be expressed as

$$
F_{\underline{\Phi}}(z)=\left.\frac{1}{1-\exp (-1)}\left[1-\exp \left(-\left\{1-\exp \left[-\boldsymbol{w}_{b, c}(z)\right]\right\}^{a}\right)\right]\right|_{z \in \mathcal{R}}
$$

where $\underline{\Phi}=(a, b, c)$ is the parameter vector of the QPGW-E model and $\mathcal{W}_{b, c}(z)=[\exp (c z)-1]^{b}$.

$i$. For $b=1$, the QPGW-E reduces to the quasi-Poisson generalized exponential-exponential distribution.

ii. For $b=2$, QPGW-E distribution reduces to quasi-Poisson generalized Rayleigh-exponential distribution.

iii. For $a=1$, the QPGW-E reduces to the quasi-Poisson Weibull-exponential distribution.

iv. For $a=b=1$, the QPGW-E reduces to quasi-Poisson exponential-exponential distribution.

v. For $b=2$ and $a=1$ the QPGW-E reduces to the reduced quasi-Poisson exponential-exponential distribution.

The PDF of the QPGW-E distribution can then be derived using (4). Figure 1 gives some plots of the PDF of the QPGW-E distribution for some selected parameter values. Figure 2 gives some plots of the HRF of the QPGW-E distribution for some selected parameter values. Based on Figure 1, we note that the new PDF of the QPGW-E distribution can be "asymmetric and right skewed shape" with no peak, "asymmetric right skewed shape" with one peak, "symmetric shape" and "asymmetric left skewed shape" with one peak. 

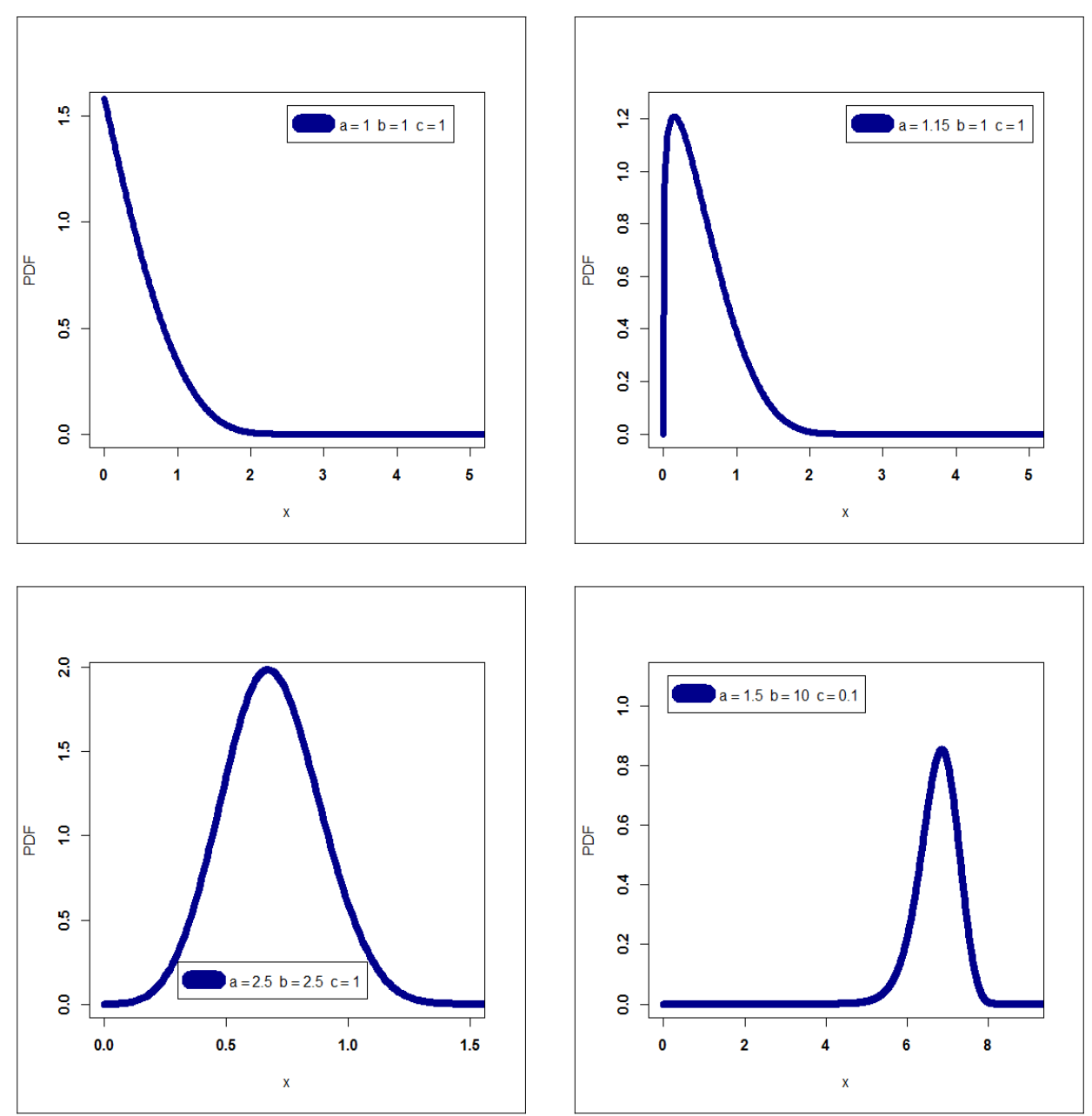

Figure 1: Some PDF plots of for the QPGW-E model. 

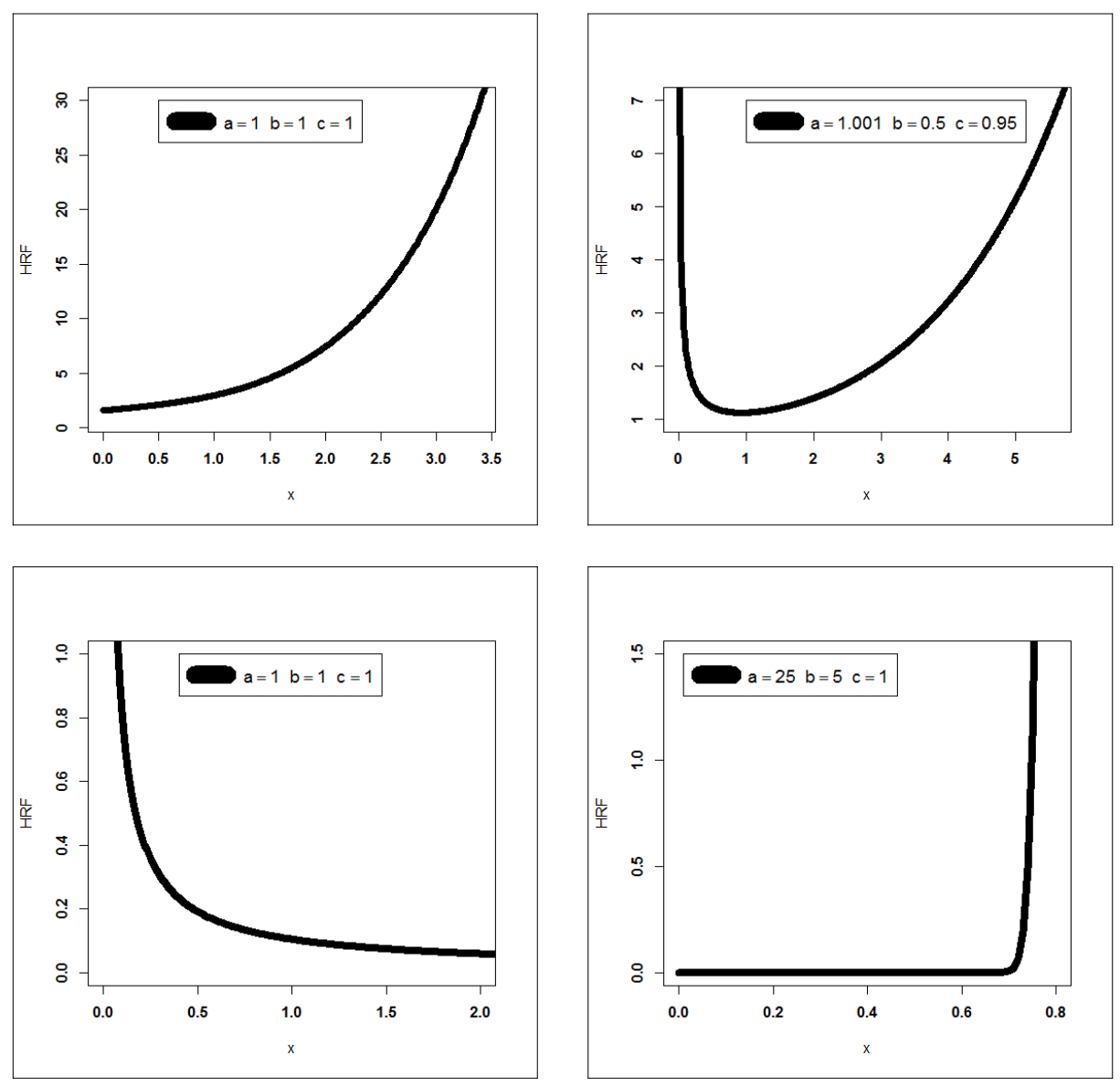

Figure 2: Some HRF plots of for the QPGW-E model.

Based on Figure 2, it is noted that the new HRF can be "increasing", "U-shape", "decreasing" and "J-shape". In the literature there are various exponential extensions which can be used in comparison such as Beta exponential (BE) model (see Lee et al. (2007)), Marshall-Olkin exponential (MO-E) extension (Ghitany et al. (2005)), Kumaraswamy exponential (Km-E) model (Cordeiro et al. (2010)), Poisson-exponential (P-E) model (Cancho et al. (2011)), Moment exponential (M-E) extension (Dara and Ahmad (2012)), Generalized Marshall-Olkin exponential (GzMO-E) model (Chakraborty and Handique (2017)), transmuted exponentiated generalized exponential (TEG-E) extension (Yousof et al. (2017a)), Marshall-Olkin Kumaraswamy exponential (MOKm-E) model (Chakraborty and Handique (2017)), Burr XII exponential (BXII-E) distribution (Cordeiro et al. (2018)), odd Lindley exponential (OL-E) extension (Almamy et al. (2018)), log Burr-Hatke exponential (LBH-E) model (Yousof et al. (2018b)), Kumaraswamy MarshallOlkin exponential (KmMO-E) distribution (George and Thobias (2019)), the Burr X exponentiated exponential (BXEE) distribution (Khalil et al. (2019)), quasi Poisson Burr X exponentiated exponential (QPBX-EE) distribution (Mansour et al. (2020a)), generalized odd log-logistic exponentiated exponential (GOLL-EE) distribution (see Mansour et al. (2020b)) and the Burr X exponential (BX-E) model (see Yousof et al. (2017b)) and Mansour et al. (2020c)), among others.

\section{The maximum likelihood method}

Let $z_{1}, z_{2}, \ldots, z_{n}$ be a random sample (RS) from the QPGW-G family with parameters $a, b, \underline{\boldsymbol{v}}^{\mathrm{T}}$ and. Let $\underline{\boldsymbol{\Phi}}=$ $\left(a, b, \underline{\mathcal{v}}^{\mathrm{T}}\right)^{\mathrm{T}}$ be the parameter vector. The log-likelihood function $\ell_{\boldsymbol{\Phi}}$ for the QPGW-G distribution is given by 


$$
\begin{aligned}
\ell_{\underline{\Phi}}= & n \log b+n \log a-n \log c_{1}+\sum_{i=1}^{n} \log \boldsymbol{h}_{\underline{v}}\left(z_{i}\right)++(b-1) \sum_{i=1}^{n} \log \mathbf{H}_{\underline{\mathcal{v}}}\left(z_{i}\right)-\sum_{i=1}^{n} \boldsymbol{W}_{b, \underline{v}}\left(z_{i}\right) \\
& -(b+1) \sum_{i=1}^{n} \overline{\mathbf{H}}_{\underline{\underline{v}}}\left(z_{i}\right)-(1-a) \sum_{i=1}^{n}\left\{1-\exp \left[-\mathcal{W}_{b, \underline{v}}\left(z_{i}\right)\right]\right\}-\sum_{i=1}^{n}\left\{1-\exp \left[-\boldsymbol{W}_{b, \underline{\mathfrak{v}}}\left(z_{i}\right)\right]\right\}^{a} .
\end{aligned}
$$

The log-likelihood function $\left(\ell_{\boldsymbol{\Phi}}\right)$ can be maximized either directly by using the SAS (PROC NLMIXED), Ox program (MaxBFGS sub-routine), R(optim function) and MATH-CAD program or by solving the nonlinear likelihood equations obtained by differentiating $\ell_{\boldsymbol{\Phi}}$. The score vector components, are given by

$$
\mathbf{U}_{a}=\frac{\partial}{\partial a} \ell_{\underline{\Phi}}, \mathbf{U}_{b}=\frac{\partial}{\partial b} \ell_{\underline{\Phi}}, \mathbf{U}_{\underline{v}_{p}}=\frac{\partial}{\partial \underline{v}_{p}} \ell_{\underline{\Phi}}
$$

Setting $\mathbf{U}_{a}=\mathbf{U}_{b}=\mathbf{U}_{\underline{v}_{p}}=0$ and solving them simultaneously yields the maximum likelihood estimations (MLEs) of $\Phi$.

\section{Applications}

In this section some competitive models are selected as competitive exponential extensions such as the odd Lindley exponential (OL-E) model, Marshall-Olkin exponential (MOE) model, Moment exponential (M-E) extension, The Logarithmic Burr-Hatke exponential (LBHE) model, Generalized Marshall-Olkin exponential (GzMO-E) model, Beta exponential (B-E) extension, Marshall-Olkin Kumaraswamy exponential (MOKm-E) model, Kumaraswamy exponential (Km-E), the Burr X exponential (BX-E) extension, Kumaraswamy Marshall-Olkin exponential (KmMOE) model and standard exponential (E) model. Some details related to these competitive extensions are available in Aboraya, M. (2019a,b and 2021b), Aboraya and Butt (2019), Elgohari and Yousof (2020), Ibrahim et al. (2020). The following are the CDFs of the competitive models:

i. $\quad$ The standard exponential model $(c>0, z>0): F_{c}(x)=1-\exp (-c z)$.

ii. $\quad$ Burr-X exponential model $(a, c>0, z>0): F_{a, c}(z)=\left(1-\exp \left\{-[\exp (c z)-1]^{2}\right\}\right)^{a}$.

iii. $\quad$ odd Lindley exponential model $(c>0, z>0)$ :

$$
F_{c}(z)=1-\frac{1+\exp (-c z)}{2 \exp (-c z)} \exp \left(\frac{-[1-\exp (-c z)]}{1-[1-\exp (-c z)]}\right) .
$$

iv. $\quad$ Kumaraswamy Marshall-Olkin exponential model $(a, b, c>0, z>0)$ :

$$
F_{a, b, \lambda, c}(z)=1-\left\{1-\left[\frac{\exp (-c z)}{1-(1-\lambda) 1-\exp (-c z)}\right]^{a}\right\}^{b} .
$$

v. Moment exponential model $(c>0, z \geq 0): F_{c}(z)=1-\left(1+\frac{z}{c}\right) e^{-\frac{z}{c}}$.

vi. $\quad$ Marshall-Olkin Kumaraswamy exponential model $(a, b, \lambda, c>0, z>0)$ :

$$
F_{a, b, \lambda, c}(z)=\frac{\left\{1-[1-\exp (-c z)]^{a}\right\}^{b}}{1-(1-\lambda)\left(1-\left\{1-[1-\exp (-c z)]^{a}\right\}^{b}\right)} .
$$

vii. Burr-Hatke exponential model $(c>0, z>0): F_{c}(z) \frac{1-\exp (-c z)}{1-c z}$.

viii. Beta exponential model $(a, b, \lambda, c>0, z>0): F_{a, b, c}(z)=I_{1-\exp (-c z)}(a, b)$.

ix. $\quad$ Marshall-Olkin exponential model $(a, c>0, z>0)$ :

$$
F_{\mathrm{a}, c}(z)=\frac{\exp (-c z)}{1-(1-\mathrm{a})[1-\exp (-c z)]}
$$

x. $\quad$ Kumaraswamy exponential model $(a, b, c>0, z>0)$ :

$$
F_{a, b, c}(z)=1-\left\{1-[1-\exp (-c z)]^{a}\right\}^{b} .
$$

xi. Generalized Marshall-Olkin exponential model $(a, b, c>0, z>0)$ :

$$
F_{\lambda, c}(z)=\frac{1-[1-\exp (-c z)]^{a}}{1-(1-b)[1-\exp (-c z)]^{a}}
$$

The following statistical tests are considered the two applications:
$i$ Cramér-von Mises (CvM-C),
ii. Anderson-Darling (AD-C),
iii. Akaike information (AI-C), 


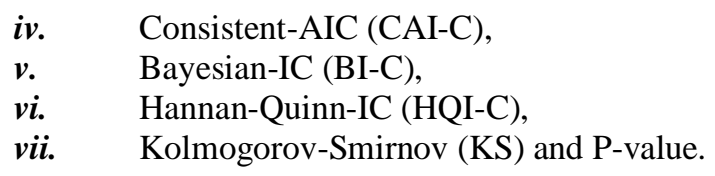

\subsection{Modeling failure (relief) times}

The first data set is related to Gross and Clark (1975) and called the failure time data. The data represents the lifetime observations relating to relief times (in minutes) of patients receiving an analgesic. The Gross and Clark data is recently analyzed by Al-Babtain et al. (2020) and Ibrahim et al. (2020). Table 1 below gives the MLEs, standard errors (SE(s)) and corresponding confidence intervals (C.I.s) for the Gross and Clark data. Table 2 below provides the AIC, BI-C, CAI-C, HQI-C, AD - C, CvM-C, K.S. and p-value for the Gross and Clark data. Figure 3 gives the box plot (top left), quantile- quantile plot (top right), total time in test (TTT) plot (bottom left) and non-parametric Kernel density estimation (NKDE) plot (bottom right) for the relief times data. Based on Figure 3 (top left and top right), the relief times data has one outlier observation. Based on Figure 3 (bottom left), the HRF of the relief times is "monotonically increasing HRF".
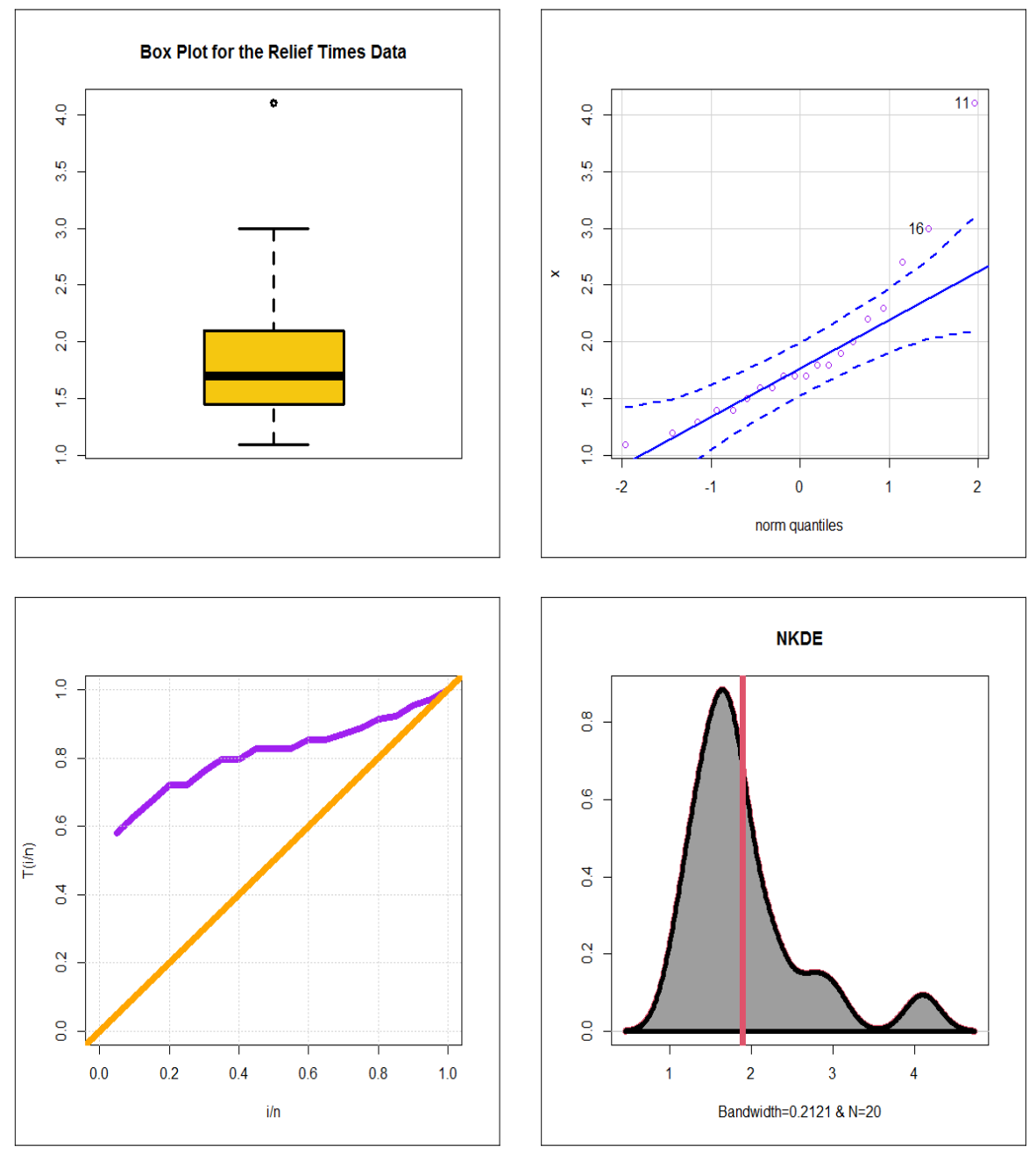

Figure 3: Plots for exploring failure (relief) times data. 
Table 1: Estimation results for the relief times data.

\begin{tabular}{|c|c|c|}
\hline $\begin{array}{c}\text { Models } \downarrow \\
\text { Estimates } \rightarrow\end{array}$ & & Estimates \\
\hline \multirow[t]{2}{*}{$\mathrm{E}(c)$} & MLEs & 0.5261 \\
\hline & $\mathrm{SE}(\mathrm{s})$ & $(0.1172)$ \\
\hline \multirow[t]{2}{*}{$\operatorname{ME}(c)$} & MLEs & 0.950 \\
\hline & $\mathrm{SE}(\mathrm{s})$ & $(0.150)$ \\
\hline \multirow[t]{2}{*}{$\operatorname{LBHE}(c)$} & MLEs & 0.5263 \\
\hline & $\mathrm{SE}(\mathrm{s})$ & $(0.118)$ \\
\hline \multirow[t]{2}{*}{$\mathrm{OL}-\mathrm{E}(c)$} & MLEs & 0.6044 \\
\hline & $\mathrm{SE}(\mathrm{s})$ & $(0.0535)$ \\
\hline \multirow[t]{2}{*}{$\mathrm{MO}-\mathrm{E}(a, c)$} & MLEs & $54.47,2.32$ \\
\hline & $\mathrm{SE}(\mathrm{s})$ & $(35.58),(0.37)$ \\
\hline \multirow[t]{2}{*}{$\mathrm{BX}-\mathrm{E}(a, c)$} & MLEs & $1.1635,0.3207$ \\
\hline & $\mathrm{SE}(\mathrm{s})$ & $(0.332),(0.033)$ \\
\hline \multirow[t]{2}{*}{$\operatorname{KmE}(a, b, c)$} & MLEs & $83.756,0.568,3.330$ \\
\hline & $\mathrm{SE}(\mathrm{s})$ & $(42.361),(0.326),(1.188)$ \\
\hline \multirow[t]{2}{*}{$\operatorname{KmMO}-\mathrm{E}(a, b, \lambda, c)$} & MLEs & $8.868,34.826,0.299,4.899$ \\
\hline & $\mathrm{SE}(\mathrm{s})$ & $(9.15),(22.31),(0.24),(3.18)$ \\
\hline \multirow[t]{2}{*}{$\operatorname{GzMO}-\mathrm{E}(a, b, c)$} & MLEs & $0.519,89.462,3.169$ \\
\hline & $\mathrm{SE}(\mathrm{s})$ & $(0.256),(66.278),(0.77)$ \\
\hline \multirow[t]{2}{*}{$\operatorname{MOKm}-\mathrm{E}(a, b, \lambda, c)$} & MLEs & $0.133,33.232,0.571,1.669$ \\
\hline & $\mathrm{SE}(\mathrm{s})$ & $(0.332),(57.84),(0.72),(1.81)$ \\
\hline \multirow[t]{2}{*}{$\mathrm{BE}(a, b, c)$} & MLEs & $81.633,0.542,3.514$ \\
\hline & $\mathrm{SE}(\mathrm{s})$ & $(120.41),(0.327),(1.410)$ \\
\hline \multirow[t]{2}{*}{ QPGW-E $(a, b, c)$} & MLEs & $29.392,0.7174,1.837$ \\
\hline & $\mathrm{SE}(\mathrm{s})$ & $(37.72),(0.688),(1.772)$ \\
\hline
\end{tabular}

Table 2: Statistics for the relief times data.

\begin{tabular}{cccccc}
\hline $\begin{array}{c}\text { Models } \downarrow \\
\text { Statistic } \rightarrow\end{array}$ & AI-C, BI-C, CAI-C, HQI-C & KS & p-value & AD-C & CvMC \\
\hline E & $68.0,68.7,67.9,68.0$ & 0.4 & 0.004 & 4.60 & 0.96 \\
BX-E & $48.1,50.1,49.0,48.5$ & 0.25 & 0.17 & 1.34 & 0.24 \\
OL-E & $49.1,50.1,49.3,49.3$ & 0.92 & $<0.01 \%$ & 1.30 & 0.22 \\
KMO-E & $43.0,46.8,45.6,43.6$ & 0.15 & 0.86 & 1.08 & 0.19 \\
M-E & $54.3,55.3,54.5,54.5$ & 0.32 & 0.07 & 2.76 & 0.53 \\
MOKm-E & $41.6,45.5,44.3,42.3$ & 0.14 & 0.87 & 0.60 & 0.11 \\
LBH-E & $67.7,68.7,67.9,67.8$ & 0.43 & $<0.01 \%$ & 0.62 & 0.11 \\
B-E & $43.5,46.5,44.9,44.0$ & 0.16 & 0.80 & 0.70 & 0.12 \\
MO-E & $43.5,45.5,44.2,43.9$ & 0.18 & 0.55 & 0.80 & 0.14 \\
Km-E & $42.0,44.8,43.3,42.3$ & 0.14 & 0.86 & 0.45 & 0.07 \\
GzMO-E & $42.8,45.7,44.3,43.3$ & 0.15 & 0.78 & 0.51 & 0.08 \\
QPGW-E & $\mathbf{3 8 . 5 , ~ 4 1 . 4 , ~ 3 9 . 9 , 3 9 . 1}$ & $\mathbf{0 . 1 3 7}$ & $\mathbf{0 . 8 4 6 9}$ & $\mathbf{0 . 3 1 9}$ & $\mathbf{0 . 0 5 3 9}$ \\
\hline
\end{tabular}



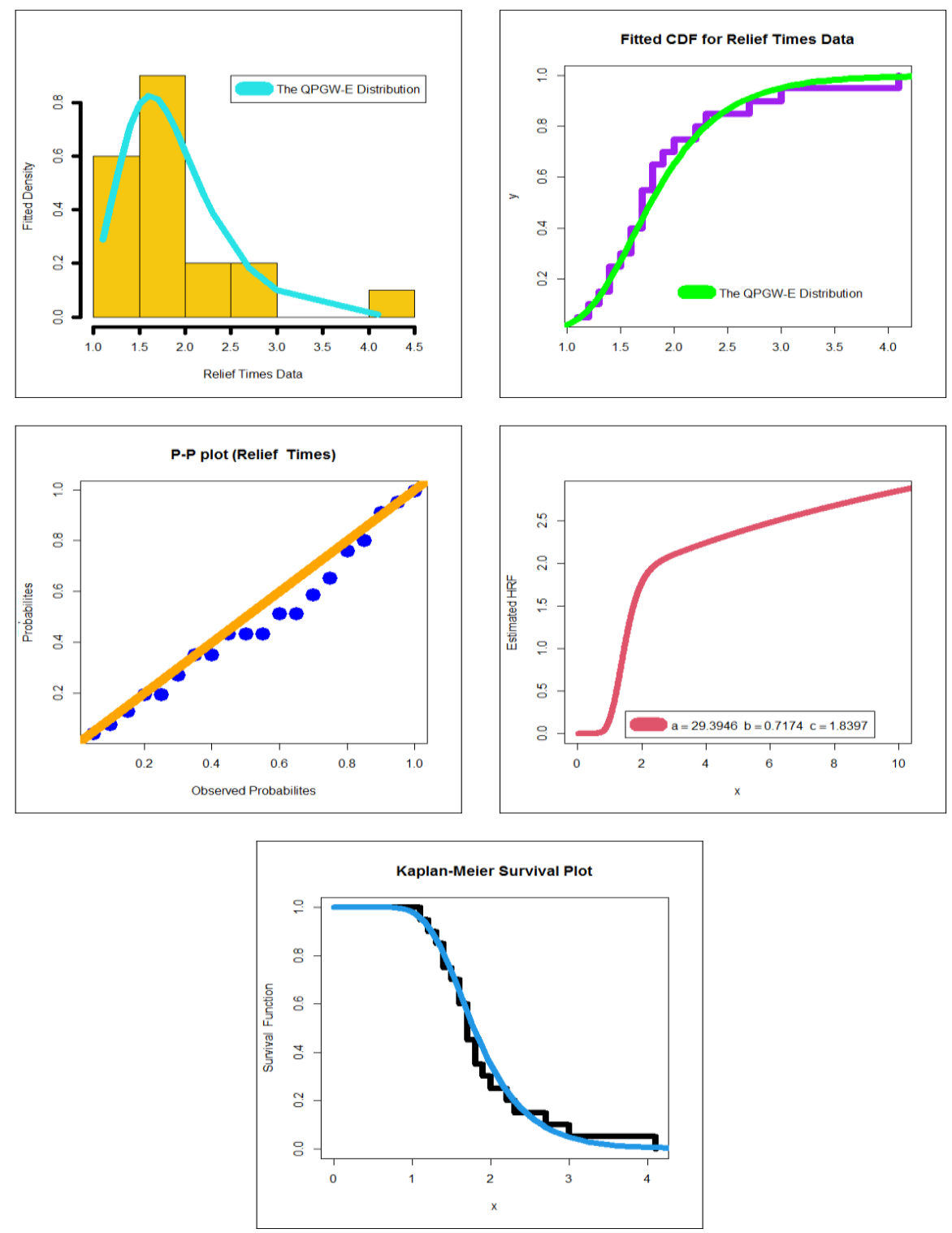

Figure 4: Fitted density, fitted CDF, P-P plot, estimated HRF and fitted survival function for relief data.

Based on Figure 3 (bottom right), NKDE of the relief times data bimodal and right skewed. Figure 4 gives the fitted density, fitted CDF, P-P plot, estimated HRF and fitted survival function for relief data. Based Figure 4, it is noted that the QPGW-E model provides adequate fits to the relief data. Based on results of Table 2, we see that the QPGWE lifetime model is better than the exponential, Odd Lindley exponential, Marshall-Olkin exponential, Moment exponential, The Logarithmic Burr-Hatke exponential, generalized Marshall-Olkin exponential, Beta exponential, Marshall-Olkin Kumaraswamy exponential, Kumaraswamy exponential, the Burr X exponential and Kumaraswamy Marshall-Olkin exponential models with $\mathrm{AI}-\mathrm{C}=38.50, \mathrm{BI}-\mathrm{C}=41.4, \mathrm{CAI}-\mathrm{C}=39.99, \mathrm{HQI}-\mathrm{C}=39.08, \mathrm{AD}-$ $\mathrm{C}=0.319, \mathrm{CvM}-\mathrm{C}=0.0539, \mathrm{KS}=0.137$ and $\mathrm{p}$-value $=0.8469$ so the new lifetime model is a good alternative to these models in modeling relief times data set.

\subsection{Modeling survival times}

The second data set called the survival times (in days) of 72 guinea pigs infected with virulent tubercle bacilli, observed and reported by Bjerkedal (1960). This data was recently analyzed by Ibrahim et al. (2020) and Al-Babtain et al. (2020). Table 3 below gives the MLEs, SE(s) and corresponding confidence intervals (C.I.s) for the guinea pigs data. 
Table 2 below provides the AI-C, BI-C, CAI-C, HQI-C, AD - C, CvM-C, K.S. and p-value for the guinea pigs data. Figure 5 gives the box plot (top left), quantile- quantile plot (top right), the TTT plot (bottom left) and the NKDE plot (bottom right) for the survival times data. Based on Figure 5 (top left and top right), the survival times data has some outlier observations. Based on Figure 5 (bottom left), the HRF of the survival times is "monotonically increasing HRF". Based on Figure 5 (bottom right), NKDE of the survival times data bimodal and right skewed. Figure 6 gives the fitted density, fitted CDF, P-P plot, estimated HRF and fitted survival function for survival data. Based Figure 6, it is noted that the QPGW-E model provides adequate fits to the survival data.

Based on results of Table 5, it is concluded that the QPGW-E lifetime model is better than the exponential, Odd Lindley exponential, Marshall-Olkin exponential, Moment exponential, The Logarithmic Burr-Hatke exponential, generalized Marshall-Olkin exponential, Beta exponential, Marshall-Olkin Kumaraswamy exponential, Kumaraswamy exponential, the Burr $\mathrm{X}$ exponential and Kumaraswamy Marshall-Olkin exponential models with $\mathrm{AI}-\mathrm{C}=204.59, \quad \mathrm{BI}-\mathrm{C}=211.43, \mathrm{CAI}-\mathrm{C}=204.95, \mathrm{HQI}-\mathrm{C}=207.31, \mathrm{AD}-\mathrm{C}=0.50, \mathrm{CvM}-\mathrm{C}=0.077$, $\mathrm{KS}=0.08572$ and $\mathrm{p}$-value $=0.6653$ so the new lifetime model is a good alternative to these models in modeling relief times data set.
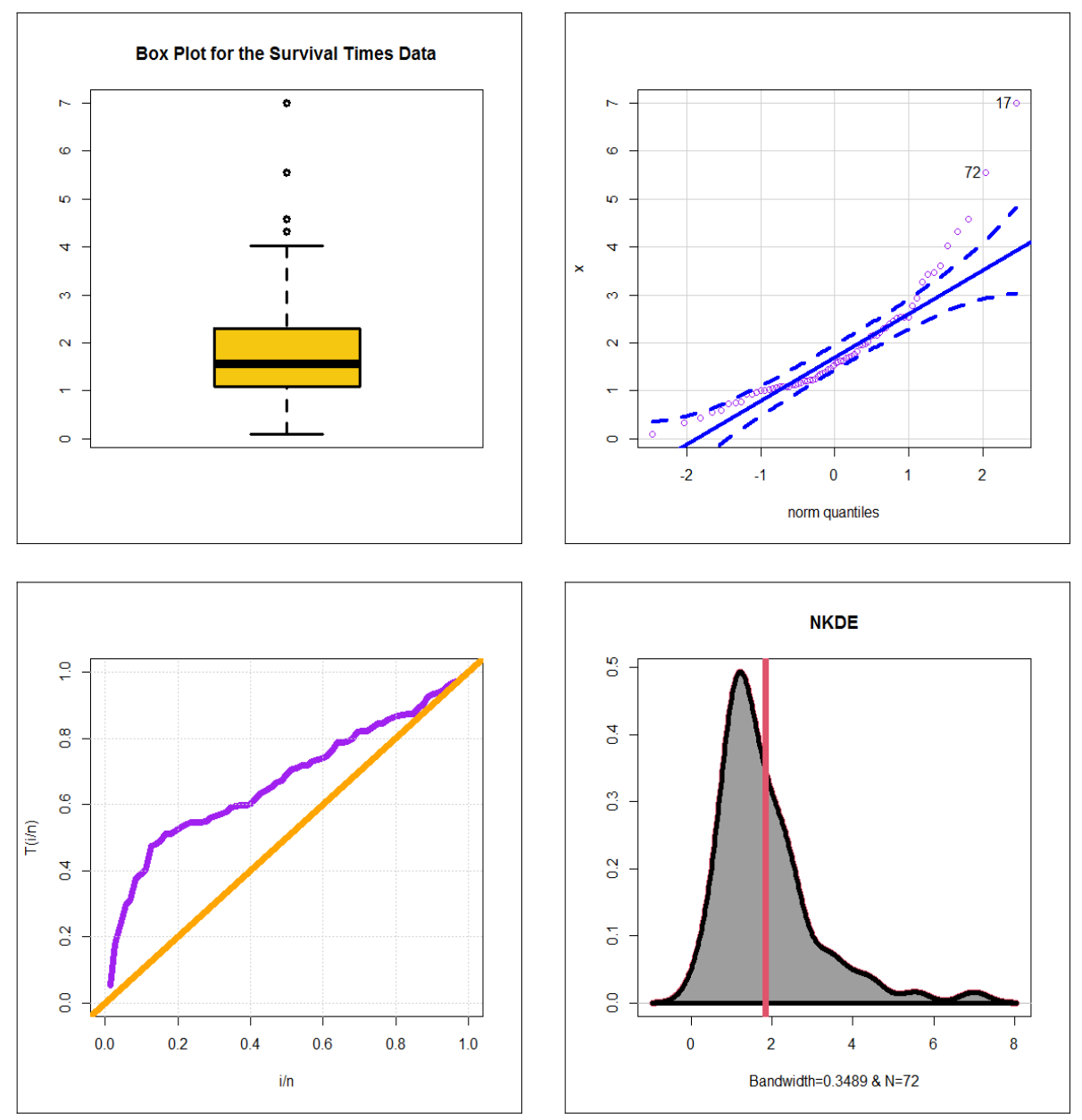

Figure 5: Plots for exploring survival times data. 
Table 3: Estimation results for the survival times data.

\begin{tabular}{|c|c|c|}
\hline $\begin{array}{c}\text { Models } \downarrow \\
\text { Estimates } \rightarrow\end{array}$ & & Estimates \\
\hline \multirow[t]{2}{*}{$\mathrm{E}(c)$} & MLEs & 0.540 \\
\hline & $\mathrm{SE}(\mathrm{s})$ & $(0.063)$ \\
\hline \multirow[t]{2}{*}{$\mathrm{OL}-\mathrm{E}(c)$} & MLEs & 0.38145 \\
\hline & $\mathrm{SE}(\mathrm{s})$ & $(0.021)$ \\
\hline \multirow[t]{2}{*}{$\operatorname{ME}(c)$} & MLEs & 0.9250 \\
\hline & $\mathrm{SE}(\mathrm{s})$ & $(0.080)$ \\
\hline \multirow[t]{2}{*}{$\mathrm{BX}-\mathrm{E}(a, c)$} & MLEs & $0.480,0.2060$ \\
\hline & $\mathrm{SE}(\mathrm{s})$ & $(0.0611),(0.012)$ \\
\hline \multirow[t]{2}{*}{$\operatorname{LBHE}(c)$} & MLEs & 0.542 \\
\hline & $\mathrm{SE}(\mathrm{s})$ & $(0.06)$ \\
\hline \multirow[t]{2}{*}{ GzMO-E $(a, b, c)$} & MLEs & $0.179,47.635,4.470$ \\
\hline & $\mathrm{SE}(\mathrm{s})$ & $(0.072),(44.901),(1.327)$ \\
\hline \multirow[t]{2}{*}{$\operatorname{MO}-\mathrm{E}(a, c)$} & MLE & $8.780,1.380$ \\
\hline & $\mathrm{SE}(\mathrm{s}) \mathrm{s}$ & $(3.555),(0.193)$ \\
\hline \multirow[t]{2}{*}{$\mathrm{B}-\mathrm{E}(a, b, c)$} & MLEs & $0.807,3.461,1.331$ \\
\hline & $\mathrm{SE}(\mathrm{s})$ & $(0.696),(1.003),(0.855)$ \\
\hline \multirow[t]{2}{*}{$\mathrm{Km}-\mathrm{E}(a, b, c)$} & MLEs & $3.3042,1.1010,1.0372$ \\
\hline & $\mathrm{SE}(\mathrm{s})$ & $(1.106),(0.764),(0.614)$ \\
\hline \multirow[t]{2}{*}{$\operatorname{KmMO}-\mathrm{E}(a, b, \lambda, c)$} & MLEs & $0.372,3.483,3.31,0.302$ \\
\hline & $\mathrm{SE}(\mathrm{s})$ & $(0.14),(0.86),(0.78),(1.11)$ \\
\hline \multirow[t]{2}{*}{$\operatorname{MOKm}-\mathrm{E}(a, b, \lambda, c)$} & MLEs & $0.0082,2.716,1.986,0.099$ \\
\hline & $\mathrm{SE}(\mathrm{s})$ & $(0.002), 1.316),(0.784),(0.048)$ \\
\hline \multirow[t]{2}{*}{$\mathrm{QPGW}-\mathrm{E}(a, b, c)$} & MLEs & $2.432,1.316,0.772$ \\
\hline & $\mathrm{SE}(\mathrm{s})$ & $(1.807),(0.638),(0.261)$ \\
\hline
\end{tabular}

Table 5: Statistic for the survival times data.

\begin{tabular}{cccccc}
\hline $\begin{array}{c}\text { Models } \downarrow \\
\text { Statistic } \rightarrow\end{array}$ & AI-C, BI-C, CAI-C, HQI-C & KS & p-value & AD-C & CvMC \\
E & $234.6,236.9,234.7,235.5$ & 0.27 & 0.060 & 6.53 & 1.25 \\
BX-E & $235.3,239.9,235.5,237.1$ & 0.22 & 0.002 & 2.90 & 0.52 \\
OL-E & $229.1,231.4,229.2,230.0$ & 0.49 & $<0.01$ & 1.94 & 0.33 \\
KMO-E & $208.0,217.0,208.4,211.4$ & 0.09 & $0.530)$ & 0.61 & 0.11 \\
M-E & $210.4,212.7,210.5,211.3$ & 0.14 & 0.130 & 1.52 & 0.25 \\
B-E & $207.4,214.2,207.7,210.1$ & 0.11 & 0.340 & 0.98 & 0.15 \\
LBH-E & $235.0,237.0,235.0,236.0$ & 0.28 & $<0.01 \%$ & 0.71 & 0.12 \\
MOKm-E & $209.4,218.6,210.0,213.0$ & 0.10 & 0.440 & 0.79 & 0.12 \\
MO-E & $210.4,215.0,210.5,212.2$ & 0.10 & 0.430 & 1.20 & 0.17 \\
Km-E & $209.4,216.2,209.8,212.1$ & 0.09 & 0.500 & 0.74 & 0.11 \\
GzMO-E & $210.5,217.4,211.0,213.2$ & 0.09 & 0.510 & 1.02 & 0.16 \\
QPGW-E & $\mathbf{2 0 4 . 6 , 2 1 1 . 4 , ~ 2 0 5 . 0 , ~ 2 0 7 . 3}$ & $\mathbf{0 . 0 8 5 7}$ & $\mathbf{0 . 6 6 5}$ & $\mathbf{0 . 5 0}$ & $\mathbf{0 . 0 7 7}$ \\
\hline
\end{tabular}



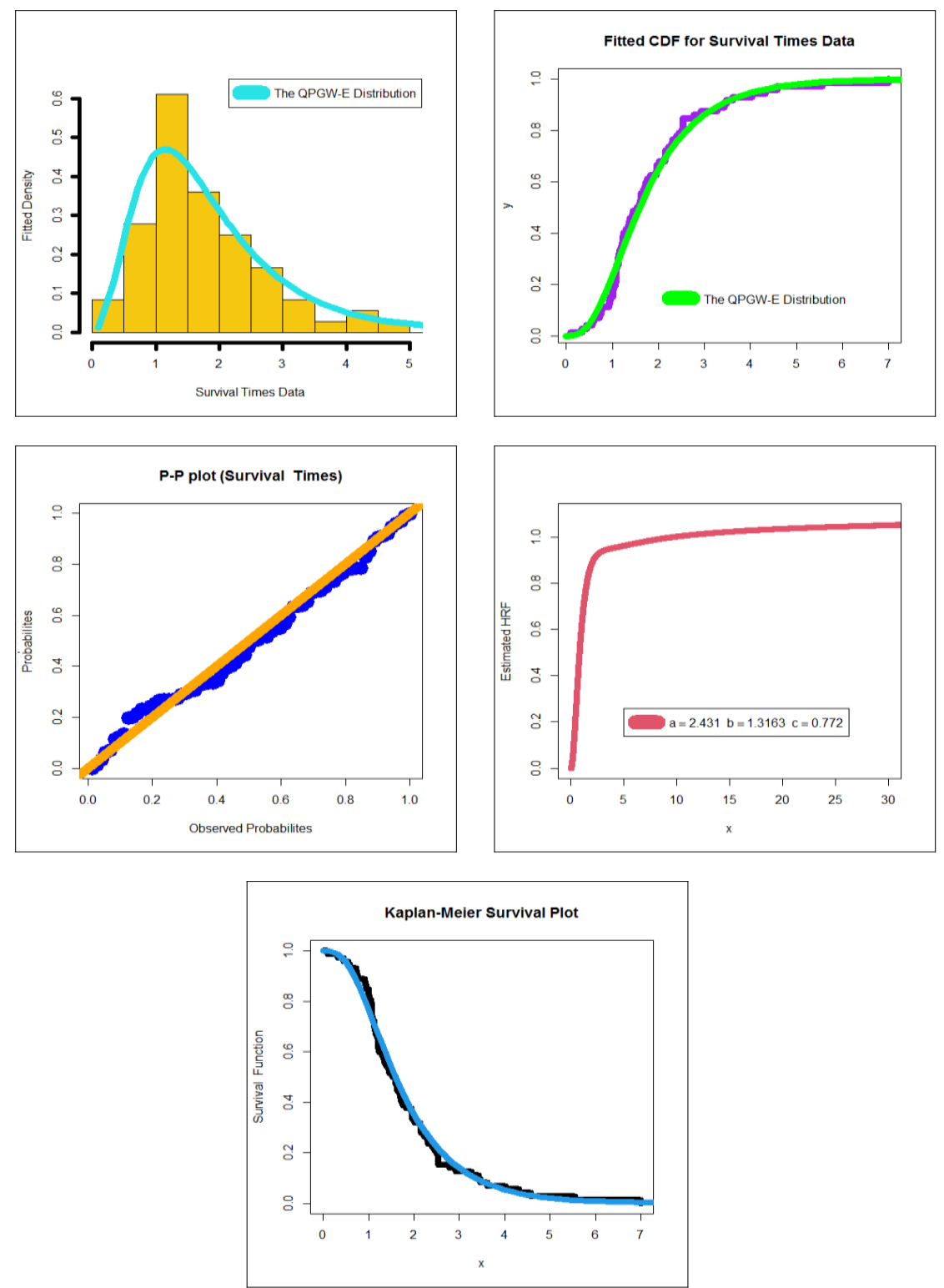

Figure 6: Fitted density, fitted CDF, P-P plot, estimated HRF and fitted survival function for survival data.

\section{Conclusions}

A novel two-parameter compound $\mathrm{G}$ family of distributions is derived and studied. Relevant statistical properties such as the ordinary moments, incomplete moments and moment generating function are derived. Using common copulas such as "Farlie-Gumbel-Morgenstern copula", "Ali-Mikhail-Haq copula", "Clayton copula" and "Renyi copula", some new bivariate type $\mathrm{G}$ families are derived. A special attention is devoted to the quasi-Poisson generalized Weibull-exponential distribution as a special case.

The density of the quasi-Poisson generalized Weibull-exponential distribution can be "asymmetric and right skewed shape" with no peak, "asymmetric right skewed shape" with one peak, "symmetric shape" and "asymmetric left skewed shape" with one peak. 
The hazard rate of the quasi-Poisson generalized Weibull-exponential distribution can be "increasing", "U-shape", "decreasing" and "J-shape". The usefulness and flexibility of the quasi-Poisson generalized Weibull-exponential distribution is illustrated by means of two applications to real data sets.

The new the quasi-Poisson generalized Weibull-exponential distribution is much better than many common exponential extensions in modeling relief times and survival times data sets under the eight criteria called AndersonDarling Criteria, Akaike Information Criteria, Cramér-Von Mises Criteria, Hannan-Quinn Information Criteria, Bayesian Information Criteria, Consistent Akaike Information Criteria, Kolmogorov-Smirnov (KS) statistic test and its corresponding p-value. As a future interesting works, many new statistic tests can be used for right censored validation such as the Nikulin Rao Robson (N.R.R) goodness-of-fit statistic test and Bagdonavicius-Nikulin (Bag.N) goodness-of-fit statistic test test (see Goual et al. (2019, 2020), Yadav et al. (2020), Ibrahim et al. (2019), Mansour et al. (2020 d-f), and Goual and Yousof (2020)). Results of characterization and regression models can be derived based on the new family (see Altun et al. (2018a-d) for more details).

\section{REFERENCES}

1. Aboraya, M. (2019a). A New Extension of the Lomax Distribution with Properties and Applications to Failure Times Data. Pakistan Journal of Statistics and Operation Research (15)2, 461-479.

2. Aboraya, M. (2019b). The extended generalized inverse Weibull distribution and its applications, Journal of Statistics and Applications, 2(1), 1-17.

3. Aboraya, M. (2021a). A new one-parameter G family of compound distributions: copulas, statistical properties and applications. Statistics, Optimization \& Information Computing, forthcoming.

4. Aboraya, M. and Butt, N. S. (2019). Extended Weibull Burr XII Distribution: Properties and Applications. Pakistan Journal of Statistics and Operation Research, (15)4, 891-903.

5. Aboraya, M. (2021b). Marshall-Olkin Lehmann Lomax Distribution: Theory, Statistical Properties, Copulas and Real Data Modeling. Pakistan Journal of Statistics and Operation Research, 17(2), 509-530.

2. Al-babtain, A. A., Elbatal, I. and Yousof, H. M. (2020). A New Flexible Three-Parameter Model: Properties, Clayton Copula, and Modeling Real Data. Symmetry, 12(3), 440.

3. Ali, M. M., Ibrahim, M. and Yousof, H. M. (2021a). Expanding the Burr X Model: Properties, Copula, Real Data Modeling and Different Methods of Estimation. OPTIMAL DECISION MAKING IN OPERATIONS RESEARCH \& STATISTICS: METHODOLOGIES AND APPLICATIONS, CRC Press, To appear.

4. Ali, M. M., Mikhail, N. N. and Haq, M. S. (1978). A class of bivariate distributions including the bivariate logistic. Journal of multivariate analysis, 8(3), 405-412.

5. Ali, M. M., Yousof, H. M. and Ibrahim, M. A New Version of the Generalized Rayleigh Distribution with Copula, Properties, Applications and Different Methods of Estimation. (2021b) OPTIMAL DECISION MAKING IN OPERATIONS RESEARCH \& STATISTICS: METHODOLOGIES AND APPLICATIONS, CRC Press, To appear.

6. Alizadeh, M., Yousof, H. M. Rasekhi, M. and Altun, E. (2019). The odd log-logistic Poisson-G Family of distributions, Journal of Mathematical Extensions, 12(3), 81-104.

7. Altun, E., Yousof, H. M. and Hamedani G. G. (2018a). A Flexible Extension of Generalized Half-Normal Distribution: Characterizations and Regression Models. International Journal of Applied Mathematics and Statistics, 57(3), 27-49.

8. Altun, E., Yousof, H. M. and Hamedani G. G. (2018b). A new flexible extension of the generalized half-normal lifetime model with characterizations and regression modeling. Bulletin of Computational Applied Mathematics, 6(1), 83-115.

9. Altun, E., Yousof, H. M. and Hamedani, G. G. (2018c). A new generalization of generalized half-normal distribution: properties and regression models. Journal of Statistical Distributions and Applications, 5(1), 7.

10. Altun, E., Yousof, H. M. and Hamedani, G. G. (2018d). A new log-location regression model with influence diagnostics and residual analysis. Facta Universitatis, Series: Mathematics and Informatics, 33(3), 417-449.

11. Almamy, J. A., Ibrahim, M., Eliwa, M. S., Al-mualim, S. and Yousof, H. M. (2018). The two-parameter odd Lindley Weibull lifetime model with properties and applications. International Journal of Statistics and Probability, 7(4), 1927-7040.

12. Aryal, G. R. and Yousof, H. M. (2017). The exponentiated generalized-G Poisson family of distributions. Economic Quality Control, 32(1), 1-17.

13. Bjerkedal, T. (1960). Acquisition of resistance in Guinea pigs infected with different doses of virulent tubercle bacilli. American Journal of Hygiene, vol. 72, 130-148.

14. Brito, E., Cordeiro, G. M., Yousof, H. M., Alizadeh, M. and Silva, G. O. (2017). The Topp-Leone odd log-logistic family of distributions. Journal of Statistical Computation and Simulation, 87(15), 3040-3058. 
15. Cancho, V. G., Louzada-Neto, F. and Barriga, G. D. (2011). The Poisson-exponential lifetime distribution. Computational Statistics \& Data Analysis, 55(1), 677-686.

16. Chakraborty, S. and Handique, L. (2017). The generalized Marshall-Olkin-Kumaraswamy-G family of distributions. Journal of data Science, 15(3), 391-422.

17. Cordeiro, G.M., Orgeta E.M.M., Nadarajah, S. (2010). The Kumaraswamy Weibull distribution with application to failure data. J. Frankl Inst, 347, 1399-1429.

18. Cordeiro, G. M., Yousof, H. M., Ramires, T. G. and Ortega, E. M. (2018). The Burr XII system of densities: properties, regression model and applications. Journal of Statistical Computation and Simulation, 88(3), 432-456.

19. Dara, S.T. and Ahmad, M. (2012). Recent Advances in Moment Distributions and their Hazard Rate. Ph.D. Thesis. National College of Business Administration and Economics, Lahore, Pakistan.

20. Elgohari, H. and Yousof, H. (2020). New Extension of Weibull Distribution: Copula, Mathematical Properties and Data Modeling. Statistics, Optimization \& Information Computing, 8(4), 972-993. https://doi.org/10.19139/soic-2310-5070-1036

21. El-Morshedy, M., Alshammari, F. S., Hamed, Y. S., Eliwa, M. S., Yousof, H. M. (2021). A New Family of Continuous Probability Distributions. Entropy, 23, 194. https://doi.org/10.3390/e23020194

22. Farlie, D. J. G. (1960). The performance of some correlation coefficients for a general bivariate distribution. Biometrika, 47, 307-323.

23. George, R. and Thobias, S. (2019). Kumaraswamy Marshall-Olkin exponential distribution. Communications in Statistics-Theory and methods, 48(8), 1920-1937.

24. Ghitany, M.E., Al-Hussaini, E.K., AlJarallah, R. A. (2005). Marshall-Olkin extended Weibull distribution and its application to censored data. J Appl Stat, 32:1025-1034.

25. Goual, H., Yousof, H. M. and Ali, M. M. (2019). Validation of the odd Lindley exponentiated exponential by a modified goodness of fit test with applications to censored and complete data. Pakistan Journal of Statistics and Operation Research, 15(3), 745-771.

26. Goual, H. and Yousof, H. M. (2020). Validation of Burr XII inverse Rayleigh model via a modified chi-squared goodness-of-fit test. Journal of Applied Statistics, 47(3), 393-423.

27. Goual, H., Yousof, H. M. and Ali, M. M. (2020). Lomax inverse Weibull model: properties, applications, and a modified Chi-squared goodness-of-fit test for validation. Journal of Nonlinear Sciences \& Applications (JNSA), 13(6), 330-353.

28. Gross, J. and Clark, V. A. (1975). Survival Distributions: Reliability Applications in the Biometrical Sciences, John Wiley, New York, USA.

29. Gumbel, E. J. (1960). Bivariate exponential distributions. Journal Amer. Statist. Assoc., 55, 698-707.

30. Gumbel, E. J. (1961). Bivariate logistic distributions. Journal of the American Statistical Association, 56(294), 335-349.

31. Hamedani, G. G., Altun, E, Korkmaz, M. C., Yousof, H. M. and Butt, N. S. (2018). A new extended G family of continuous distributions with mathematical properties, characterizations and regression modeling. Pak. J. Stat. Oper. Res., 14(3), 737-758.

32. Hamedani, G. G. Rasekhi, M., Najib, S. M., Yousof, H. M. and Alizadeh, M., (2019). Type II general exponential class of distributions. Pak. J. Stat. Oper. Res., XV (2), 503-523.

33. Hamedani, G. G. Yousof, H. M., Rasekhi, M., Alizadeh, M., Najibi, S. M. (2017). Type I general exponential class of distributions. Pak. J. Stat. Oper. Res., XIV (1), 39-55.

34. Ibrahim, M., Altun, E. and Yousof, H. M. (2020). A new distribution for modeling lifetime data with different methods of estimation and censored regression modeling. Statistics, Optimization \& Information Computing, 8(2), 610-630.

35. Ibrahim, M., Yadav, A. S., Yousof, H. M., Goual, H. and Hamedani, G. G. (2019). A new extension of Lindley distribution: modified validation test, characterizations and different methods of estimation. Communications for Statistical Applications and Methods, 26(5), 473-495.

36. Johnson, N. L. and Kotz, S. (1977). On some generalized Farlie-Gumbel-Morgenstern distributions- II: Regression, correlation and further generalizations. Commun. Stat.Theory, 6, 485-496.

37. Karamikabir, H., Afshari, M., Yousof, H. M., Alizadeh, M. and Hamedani, G. (2020). The Weibull ToppLeone Generated Family of Distributions: Statistical Properties and Applications. Journal of The Iranian Statistical Society, 19(1), 121-161

38. Khalil, M. G., Hamedani, G. G. and Yousof, H. M. (2019). The Burr X exponentiated Weibull model: Characterizations, mathematical properties and applications to failure and survival times data. Pakistan Journal of Statistics and Operation Research, 141-160.

39. Korkmaz, M. C. Yousof, H. M. and Hamedani G. G. (2018a). The exponential Lindley odd log-logistic G family: 
properties, characterizations and applications. Journal of Statistical Theory and Applications, 17(3), 554 - 571

40. Korkmaz, M. C., Yousof, H. M., Hamedani G. G. and Ali, M. M. (2018b). The Marshall-Olkin generalized G Poisson family of distributions, Pakistan Journal of Statistics, 34(3), 251-267.

41. Lee, C., Famoye, F. and Olumolade, O. (2007). Beta-Weibull distribution: some properties and applications to censored data. Journal of modern applied statistical methods, 6(1), 17.

42. Mansour, M. M., Ibrahim, M., Aidi, K., Shafique Butt, N., Ali, M. M., Yousof, H. M. and Hamed, M. S. (2020a). A New Log-Logistic Lifetime Model with Mathematical Properties, Copula, Modified Goodness-of-Fit Test for Validation and Real Data Modeling. Mathematics, 8(9), 1508.

43. Mansour, M. M., Butt, N. S., Ansari, S. I., Yousof, H. M., Ali, M. M. and Ibrahim, M. (2020b). A new exponentiated Weibull distribution's extension: copula, mathematical properties and applications. Contributions to Mathematics, 1 (2020) 57-66. DOI: 10.47443/cm.2020.0018

44. Mansour, M., Korkmaz, M. C., Ali, M. M., Yousof, H. M., Ansari, S. I. and Ibrahim, M. (2020c). A generalization of the exponentiated Weibull model with properties, Copula and application. Eurasian Bulletin of Mathematics, 3(2), 84-102.

45. Mansour, M., Rasekhi, M., Ibrahim, M., Aidi, K., Yousof, H. M. and Elrazik, E. A. (2020d). A New Parametric Life Distribution with Modified Bagdonavicius-Nikulin Goodness-of-Fit Test for Censored Validation, Properties, Applications, and Different Estimation Methods. Entropy, 22(5), 592.

46. Mansour, M., Yousof, H. M., Shehata, W. A. and Ibrahim, M. (2020e). A new two parameter Burr XII distribution: properties, copula, different estimation methods and modeling acute bone cancer data. Journal of Nonlinear Science and Applications, 13(5), 223-238.

47. Mansour, M. M., Butt, N. S., Yousof, H. M., Ansari, S. I. and Ibrahim, M. (2020f). A Generalization of Reciprocal Exponential Model: Clayton Copula, Statistical Properties and Modeling Skewed and Symmetric Real Data Sets. Pakistan Journal of Statistics and Operation Research, 16(2), 373-386.

48. Merovci, F., Alizadeh, M., Yousof, H. M. and Hamedani, G. G. (2017). The exponentiated transmuted-G family of distributions: theory and applications. Communications in Statistics-Theory and Methods, 46(21), 1080010822.

49. Merovci, F., Yousof, H. M. and Hamedani, G. G. (2020). The Poisson Topp Leone Generator of Distributions for Lifetime Data: Theory, Characterizations and Applications. Pakistan Journal of Statistics and Operation Research, $16(2), 343-355$.

50. Morgenstern, D. (1956). Einfache beispiele zweidimensionaler verteilungen. Mitteilingsblatt fur Mathematische Statistik, 8, 234-235.

51. Nascimento, A. D. C., Silva, K. F., Cordeiro, G. M., Alizadeh, M. and Yousof, H. M. (2019). The odd Nadarajah-Haghighi family of distributions: properties and applications. Studia Scientiarum Mathematicarum Hungarica, 56(2), 1-26

52. Nelsen, R. B. (2007). An introduction to copulas. Springer Science \& Business Media.

53. Pougaza, D. B. and Djafari, M. A. Maximum entropies copulas. Proceedings of the 30th international workshop on Bayesian inference and maximum Entropy methods in Science and Engineering, 2011, 329-336.

54. Ramos, M. W. A., Meranho, P. R. D., Cordeiro, G. M., da Silva, R. V. and Hamedani, G. G. (2015). The Kumaraswamy-G Poisson family of distributions, J. Stat. Theory Appl., 14, 222-239.

55. Yadav, A. S., Goual, H., Alotaibi, R. M., Ali, M. M. and Yousof, H. M. (2020). Validation of the Topp-LeoneLomax model via a modified Nikulin-Rao-Robson goodness-of-fit test with different methods of estimation. Symmetry, 12(1), 57.

56. Yousof, H. M., Afify, A. Z., Alizadeh, M., Butt, N. S., Hamedani, G. G. and Ali, M. M. (2015). The transmuted exponentiated generalized-G family of distributions, Pak. J. Stat. Oper. Res., 11, 441-464.

57. Yousof, H. M., Afify, A. Z., Alizadeh, M., Hamedani G. G., Jahanshahi, S. M. A. and Ghosh, I., (2018a). The generalized transmuted Poisson-G family of Distributions, Pak. J. Stat. Oper. Res., Vol. 14 (4), pp. $759-779$.

58. Yousof, H. M., Afify, A. Z., Cordeiro, G. M., Alzaatreh, A. and Ahsanullah, M. (2017a). A new four-parameter Weibull model for lifetime data. Journal of Statistical Theory and Applications, 16(4), 448-466.

59. Yousof, H. M., Afify, A. Z., Hamedani, G. G. and Aryal, G. (2017b). The Burr X generator of distributions for lifetime data. Journal of Statistical Theory and Applications, 16(3), 288-305.

60. Yousof, H. M., Altun, E., Ramires, T. G., Alizadeh, M. and Rasekhi, M. (2018b). A new family of distributions with properties, regression models and applications. Journal of Statistics and Management Systems, 21(1), 163188.

61. Yousof, H. M., Mansoor, M. Alizadeh, M., Afify, A. Z., Ghosh, I. and Afify, A. Z. (2020). The Weibull-G Poisson family for analyzing lifetime data. Pak. J. Stat. Oper. Res., 16 (1), 131-148. 